\title{
A New Effective Side Length Expression Obtained Using a Modified Tabu Search Algorithm for the Resonant Frequency of a Triangular Microstrip Antenna
}

\author{
Derviş Karaboğa, Kerim Güney, Ahmet Kaplan, Ali Akdağli \\ Department of Electronic Engineering, Erciyes University, 38039 Kayseri, Turkey; \\ e-mail: karaboga@zirve.erciyes.edu.tr
}

Received 2 August 1996; revised 2 December 1996

\begin{abstract}
A new, very simple curve-fitting expression for the effective side length is presented for the resonant frequency of triangular microstrip antennas. It is obtained using a modified tabu search algorithm, and is useful for the computer-aided design (CAD) of microstrip antennas. The theoretical resonant frequency results obtained using this new effective side length expression are in very good agreement with the experimental results available in the literature. () 1998 John Wiley \& Sons, Inc. Int $J$ RF and Microwave CAE 8: 4-10, 1998.
\end{abstract}

Keywords: microstrip antenna; triangular; resonant frequency; optimization; effective side length; tabu search algorithm

\section{INTRODUCTION}

Microstrip antennas are among the most popular antenna types, because they are lightweight, have simple geometries, are inexpensive to fabricate, and can easily be made conformal to the host body [1-5]. The majority of the studies proposed in this area have concentrated on rectangular and circular microstrip antennas. However, it is known that the triangular patch antenna has radiation properties similar to those of the rectangular antenna, with the advantage of being physically smaller. Triangular microstrip antennas present a particular interest for the design of periodic arrays because triangular radiating elements can be arranged in a manner that allows the designer to reduce significantly the coupling between adjacent elements of the array. This significantly simplifies array design. In triangular microstrip antenna designs, it is important to determine the resonant frequencies of the antenna accurately

Correspondence to: K. Güney because microstrip antennas have narrow bandwidths and can only operate effectively in the vicinity of the resonant frequency. As such, a theory to help ascertain the resonant frequency is helpful in antenna designs.

The resonant frequency of such antennas is a function of the side length of the patch, the permittivity of the substrate, and its thickness. A number of methods [1,6-14] are available to determine the resonant frequency of an equilateral triangular microstrip patch antenna, as this is one of the most popular and convenient shapes. The experimental resonant frequency results of this antenna have been reported elsewhere [7, 11]. The theoretical resonant frequency values presented in the literature [1,6-14] are not in very good agreement with the experimental results. For this reason, a new, very simple effective side length expression is presented in this article for an equilateral triangular patch antenna. The resonant frequencies of this antenna are then obtained by using this new effective side length 
expression and the relative dielectric constant of the substrate.

In this work, first, a model for the effective side length expression is chosen, then the unknown coefficient values of the expression are obtained by a modified tabu search algorithm. The tabu search algorithm is a very efficient and flexible optimization technique developed especially for combinatorial optimization problems $[15$, 16]. But, it has also produced very good solutions for numerical optimization problems [17-19]. The tabu search algorithm used here employs an adaptive neighbor production mechanism. Therefore, this algorithm is different from the tabu search algorithms in the literature $[15,16]$.

The theoretical resonant frequency results obtained using the new, simple effective side length expression presented here are in very good agreement with the experimental results [7,11]. Güney [20-22] also proposed very simple expressions for accurately calculating the resonant frequencies of rectangular and circular microstrip antennas.

Most of the previous theoretical resonant frequency results for triangular microstrip antennas were compared only with the experimental results reported by Dahele and Lee [7]. In this work, the theoretical results obtained using the formulae available in the literature are compared with the experimental results reported by Dahele and Lee [7], and also Chen et al. [11].

\section{FORMULATION}

For a triangular microstrip antenna, the resonant frequencies obtained from the cavity model with perfect magnetic walls are given by the formula [6]:

$$
f_{m n}=\frac{2 c}{3 a\left(\epsilon_{r}\right)^{1 / 2}}\left[m^{2}+m n+n^{2}\right]^{1 / 2}
$$

where $c$ is the velocity of electromagnetic waves in free space, $\epsilon_{r}$ is the relative dielectric constant of the substrate, subscript $m n$ refers to $\mathrm{TM}_{m n}$ modes, and $a$ is the length of a side of the triangle, as shown in Figure 1.

Eq. (1) is based on the assumption of a perfect magnetic wall and neglects the fringing fields at the open-end edge of the microstrip patch. To account for these fringing fields, there are a number of suggestions [1, 6-14]. The most common suggestion is that side length $a$ in eq. (1) be replaced by an effective value $a_{\text {eff }}$. The same suggestion is also used in this study. The effective

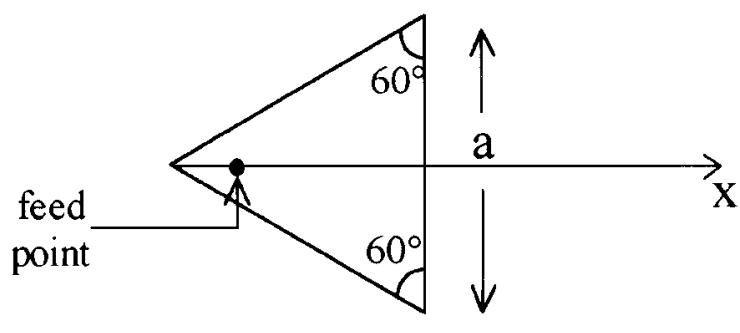

conducting

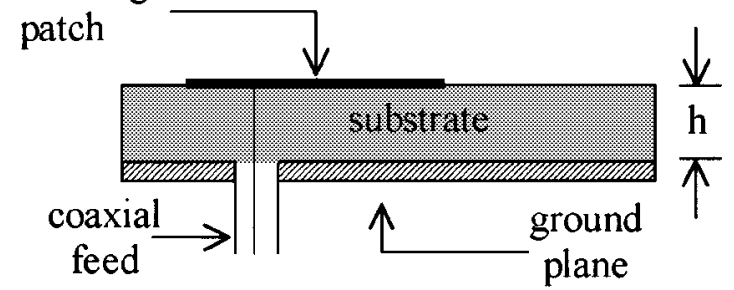

Figure 1. Geometry of equilateral triangular microstrip antenna.

side length, $a_{\text {eff }}$, which is slightly larger than the physical side length $a$, takes into account the influence of the fringing field at the edges and the dielectric inhomogeneity of the triangular microstrip patch antenna. It is clear from all of the formulae proposed [1, 6-14] that the effective side length of a triangular microstrip antenna is determined by the relative dielectric constant of the substrate, $\epsilon_{r}$, the physical side length, $a$, and the thickness of the substrate, $h$. Therefore, the effective side length expression, $a_{\text {eff }}$, to be found must be larger than $a$ and depend on $\epsilon_{r}, a$, and $h$.

The problem in the literature is that an expression that is as simple as possible for the effective side length should be obtained, but the theoretical results obtained by using the expression must be in good agreement with the experimental results. In this work, a new technique based on the tabu search algorithm for solving this problem efficiently is presented. First, a model for the effective side length expression is chosen, then the unknown coefficients of the model are determined by a modified tabu search algorithm.

To find the proper model for the effective side length expression, many experiments were carried out in this work. After many trials, the following model, depending on $\epsilon_{r}, a$, and $h$, which produces good results, was chosen:

$$
a_{\mathrm{eff}}=a+h\left(\alpha_{1}+\frac{\alpha_{2}}{\epsilon_{r}^{\alpha_{3}}}\right)
$$

where the unknown coefficients $\alpha_{1}, \alpha_{2}$, and $\alpha_{3}$ are determined by a modified tabu search algo- 
rithm. It is evident from eq. (2) that the effective side length, $a_{\text {eff }}$, is larger than the physical side length, $a$, provided $\alpha_{1}, \alpha_{2}$, and $\alpha_{3}$ are greater than zero.

The tabu search algorithm, which is based on intelligent problem solving tenets, is an optimization technique developed especially for combinatorial optimization problems, but it has also produced efficient solutions for numeric problems. It is a form of iterative search and does not use derivative-based transition rules.

The tabu search starts with an arbitrary solution created by a random number generator. In this particular problem, it is equivalent to starting with randomly generated values for the effective side length expression coefficients. A solution is represented with a vector of real numbers (coefficient values) and an associated set of neighbors. A neighbor is reached directly from the present solution by an operation called "move." A succession of moves is carried out to transform the arbitrary solution to an optimal one. The new solution is the highest evaluation move among the neighbors in terms of the performance value and tabu restrictions which exist to avoid new moves that were evaluated in earlier iterations.

The tabu search used in this work employs an adaptive mechanism for producing neighbors. The neighbors of a present solution are created by the following procedure.

If $a_{\text {eff }}(t)=\left(\alpha_{1}, \alpha_{2}, \alpha_{3}\right)$ is the solution vector at the $t$ th iteration, two neighbors $\left(a_{\text {eff }}\left(n_{1}, n_{2}\right)\right)$ of this solution of which the element $\alpha_{k}$ is not in the tabu list are produced by:

$$
a_{\mathrm{eff}}\left(\bar{n}_{1}, \bar{n}_{2}\right)= \begin{cases}\alpha_{k}+\Delta(t) & \text { for odd neighbors } \\ \alpha_{k}-\Delta(t) & \text { for even neighbors }\end{cases}
$$

$$
a_{\text {eff }}\left(n_{1}, n_{2}\right)=\operatorname{Remain}\left(a_{\text {eff }}\left(\bar{n}_{1}, \bar{n}_{2}\right), \alpha_{\text {max }}\right)
$$

with

$$
\Delta(t)=k_{1}\left[\frac{\text { LatestImprovementIteration }}{\text { Iteration }^{k_{2}}+\text { LatestImprovementIteration }}\right]^{k_{3}}
$$

where Iteration stands for the current iteration number and LatestImprovementIteration is the iteration number at which the latest improvement was obtained. The value of $\alpha_{\max }$, which is larger than zero for each coefficient, is determined after several experiments by the designer, and which is taken as 5 in this work. The index, $t$, in $\Delta(t)$ represents the iteration number. The Remain function in eq. (4) keeps the elements of the solution within the desired range. While $k_{1}$ in eq. (5) determines the magnitude of $\Delta(t), k_{2}$ and $k_{3}$ control the change of $\Delta(t)$. The proper values for the parameter $k_{1}, k_{2}$, and $k_{3}$ in eq. (5) are determined by experience on the tabu search. In the present work, the values taken for $k_{1}, k_{2}$, and $k_{3}$ are 10,2 , and 2 , respectively.

Tabu restrictions used here are based on the recency and frequency memory storing the information about the past steps of the search. The recency-based memory prevents cycles of length less than or equal to a predetermined number of iterations from occurring in the trajectory. The frequency-based memory keeps the number of change of solution vector elements. If an element of the solution vector does not satisfy the following tabu restrictions, then it is accepted as tabu:

Tabu Restrictions

$$
=\left\{\begin{array}{c}
\operatorname{recency}(k)>\text { restriction period } \\
\text { or } \\
\text { frequency }(k)<\text { frequency limit }
\end{array}\right.
$$

To select the new solution from the neighbors, evaluation values of the neighbors are calculated using their recency, frequency, and performance values.

The formula used for the evaluation of a solution is:

$$
\begin{aligned}
& \text { evaluation }(i) \\
& \qquad \begin{array}{l}
a * \operatorname{improvement}(i)+b * \operatorname{recency}(i) \\
-c * \operatorname{frequency}(i)
\end{array}
\end{aligned}
$$

where $a, b$, and $c$ are the improvement, recency, and frequency factors, and equal to 4, 2, and 1, respectively, in this study. In eq. (7) the improvement is the difference between the performance of the best solution found so far and that of the $i$ th neighbor. The performance of a neighbor can be computed using various formulas. In our work, the following is employed:

$$
P(i)=A-\sum_{j=1}^{N}\left|f(j)_{m e}-f(j)_{c a}\right|
$$

where $A$ is a positive constant selected to be large enough so that $P(i)$ values are positive for 
TABLE I. Comparison of Measured and Calculated Resonant Frequencies of the First Five Modes of an Equilateral Triangular Microstrip Antenna with $a=10 \mathrm{~cm}, \varepsilon_{r}=2.32$, and $h=0.159 \mathrm{~cm}$

\begin{tabular}{|c|c|c|c|c|c|c|c|c|c|c|c|c|}
\hline Mode & $\begin{array}{c}f_{m e} \\
{[7]} \\
(\mathrm{MHz})\end{array}$ & $\begin{array}{l}f_{d a} \\
\text { present } \\
\text { method } \\
(\mathrm{MHz})\end{array}$ & $\begin{array}{c}f_{b b} \\
{[1]} \\
(\mathrm{MHz})\end{array}$ & $\begin{array}{c}f_{h j} \\
{[6]} \\
(\mathrm{MHz})\end{array}$ & $\begin{array}{c}f_{g l} \\
{[8]} \\
(\mathrm{MHz})\end{array}$ & $\begin{array}{c}f_{g a} \\
{[9]} \\
(\mathrm{MHz})\end{array}$ & $\begin{array}{c}f_{s d} \\
{[10]} \\
(\mathrm{MHz})\end{array}$ & $\begin{array}{c}f_{c l 1} \\
\text { moment } \\
\text { method [11] } \\
(\mathrm{MHz})\end{array}$ & $\begin{array}{c}f_{c l 2} \\
{[11]} \\
(\mathrm{MHz})\end{array}$ & $\begin{array}{c}f_{g u 1} \\
{[12]} \\
(\mathrm{MHz})\end{array}$ & $\begin{array}{c}f_{k k} \\
{[14]} \\
(\mathrm{MHz})\end{array}$ & $\begin{array}{c}f_{g u 2} \\
{[13]} \\
(\mathrm{MHz})\end{array}$ \\
\hline $\mathrm{TM}_{10}$ & 1280 & 1281 & 1413 & 1299 & 1273 & 1340 & 1273 & 1288 & 1296 & 1280 & 1289 & 1280 \\
\hline $\mathrm{TM}_{11}$ & 2242 & 2218 & 2447 & 2251 & 2206 & 2320 & 2206 & 2259 & 2244 & 2217 & 2233 & 2218 \\
\hline $\mathrm{TM}_{20}$ & 2550 & 2562 & 2826 & 2599 & 2547 & 2679 & 2547 & 2610 & 2591 & 2560 & 2579 & 2561 \\
\hline $\mathrm{TM}_{21}$ & 3400 & 3389 & 3738 & 3438 & 3369 & 3544 & 3369 & 3454 & 3428 & 3387 & 3411 & 3387 \\
\hline $\mathrm{TM}_{30}$ & 3824 & 3842 & 4239 & 3898 & 3820 & 4019 & 3820 & 3875 & 3887 & 3840 & 3868 & 3841 \\
\hline
\end{tabular}

all possible solutions, which is taken as 1000 in the present work, and $f_{m e}$ and $f_{c a}$ represent, respectively, the measured resonant frequency values and the calculated resonant frequency values by using effective side length expression constructed by the modified tabu search algorithm. The measured data sets used for the optimization and evaluation process have been obtained from the previous works, which are given in Tables I-III. The fourth entries in these tables are used for the evaluation process to demonstrate the accuracy of the model and the remainder 12 data sets $[N=12$ in eq. (8)] are used for the optimization process. Only three measured data sets are used for the evaluation process because of the limited measured data available in the literature.

The unknown coefficient values of the model given in eq. (2) are optimized by the modified tabu search algorithm just described. The optimum values found are:

$$
\alpha_{1}=0.1, \quad \alpha_{2}=8, \quad \alpha_{3}=2
$$

The following effective side length expression, $a_{\text {eff }}$, is obtained by substituting the coefficient values given by eq. (9) into eq. (2).

$$
a_{\text {eff }}=a+h\left(0.1+\frac{8}{\epsilon_{r}^{2}}\right)
$$

The resonant frequencies are then calculated by the formula

$$
f_{m n}=\frac{2 c}{3 a_{\mathrm{eff}}\left(\epsilon_{r}\right)^{1 / 2}}\left[m^{2}+m n+n^{2}\right]^{1 / 2}
$$

\section{RESULTS AND DISCUSSION}

To determine the most appropriate suggestion given in the literature, we compared our com- puted values of the resonant frequencies for the first five modes of the different equilateral triangular patch antennas with the theoretical and experimental results reported by other scientists, which are all given in Tables I-III. The entries of $f_{m e}, f_{d a}, f_{b b}, f_{h j}, f_{g l}, f_{g a}, f_{s d}, f_{c l 1}, f_{c l 2}, f_{g u 1}, f_{k k}$, and $f_{g u 2}$ represent, respectively, the values measured [7, 11], calculated by this method, calculated by Bahl and Bhartia [1], calculated by Helszajn and James [6], calculated by Garg and Long [8], calculated by Gang [9], calculated by Singh et al. [10], calculated by using moment method [11], calculated by using the curve-fitting formula proposed by Chen et al. [11], calculated by Güney [12], calculated by Kumprasert and Kiranon [14], and calculated by Güney [13]. In Table I, the resonant frequencies were measured by Dahele and Lee [7]. In Tables II and III, the resonant frequencies were measured by Chen et al. [11]. The total absolute errors between the theoretical and experimental results in Tables I-III for every suggestion are also listed in Table IV.

The theoretical results predicted by Garg and Long [8], and Singh et al. [10] are the same, because the analytical formulas proposed by these scientists are the same.

In ref. 11, the moment method full-wave analysis and also the curve-fitting formula based on the data set obtained from this moment method fullwave analysis were presented for the resonant frequency of a triangular patch antenna. However, it is apparent from Tables I-IV that the theoretical resonant frequency results calculated from this curve-fitting formula and the moment method full-wave analysis are not in very good agreement with the experimental results. It is also evident from Tables I-IV that the theoretical resonant frequency results calculated from the theories available in the literature [1, 6-14] are also not in very good agreement with the experimental results. For these reasons, the data set 
TABLE II. Comparison of Measured and Calculated Resonant Frequencies of the First Five Modes of an Equilateral Triangular Microstrip Antenna with $a=8.7 \mathrm{~cm}, \varepsilon_{r}=2.32$, and $h=0.078 \mathrm{~cm}$

\begin{tabular}{|c|c|c|c|c|c|c|c|c|c|c|c|c|}
\hline Mode & $\begin{array}{c}f_{m e} \\
{[11]} \\
(\mathrm{MHz})\end{array}$ & $\begin{array}{c}f_{d a} \\
\text { present } \\
\text { method } \\
(\mathrm{MHz})\end{array}$ & $\begin{array}{c}f_{b b} \\
{[1]} \\
(\mathrm{MHz})\end{array}$ & $\begin{array}{c}f_{h j} \\
{[6]} \\
(\mathrm{MHz})\end{array}$ & $\begin{array}{c}f_{g l} \\
{[8]} \\
(\mathrm{MHz})\end{array}$ & $\begin{array}{c}f_{g a} \\
{[9]} \\
(\mathrm{MHz})\end{array}$ & $\begin{array}{c}f_{s d} \\
{[10]} \\
(\mathrm{MHz})\end{array}$ & $\begin{array}{c}f_{c l 1} \\
\text { moment } \\
\text { method [11] } \\
(\mathrm{MHz})\end{array}$ & $\begin{array}{c}f_{c l 2} \\
{[11]} \\
(\mathrm{MHz})\end{array}$ & $\begin{array}{c}f_{g u 1} \\
{[12]} \\
(\mathrm{MHz})\end{array}$ & $\begin{array}{c}f_{k k} \\
{[14]} \\
(\mathrm{MHz})\end{array}$ & $\begin{array}{c}f_{g u 2} \\
{[13]} \\
(\mathrm{MHz})\end{array}$ \\
\hline $\mathrm{TM}_{10}$ & 1489 & 1488 & 1627 & 1500 & 1480 & 1532 & 1480 & 1498 & 1498 & 1486 & 1493 & 1481 \\
\hline $\mathrm{TM}_{11}$ & 2596 & 2577 & 2818 & 2599 & 2564 & 2654 & 2564 & 2608 & 2595 & 2573 & 2585 & 2565 \\
\hline $\mathrm{TM}_{20}$ & 2969 & 2976 & 3254 & 3001 & 2961 & 3065 & 2961 & 2990 & 2996 & 2971 & 2985 & 2962 \\
\hline $\mathrm{TM}_{21}$ & 3968 & 3937 & 4304 & 3970 & 3917 & 4054 & 3917 & 3977 & 3963 & 3931 & 3949 & 3918 \\
\hline $\mathrm{TM}_{30}$ & 4443 & 4464 & 4880 & 4501 & 4441 & 4597 & 4441 & 4480 & 4494 & 4457 & 4478 & 4443 \\
\hline
\end{tabular}

obtained from the moment method and the existing theories are not used in this work. The measured data set is used only for the optimization process.

We observe that our results calculated by using $a_{\text {eff }}$ presented here are better than those predicted by other scientists. This is clear from Tables I-IV. The very good agreement between the measured values and our computed resonant frequency values supports the validity of the simple curve-fitting effective side length expression obtained using the modified tabu search algorithm, even with the limited data set. We expect that the modified tabu search algorithm will find wide application in computer-aided design (CAD) of microstrip antennas and microwave-integrated circuits. The results obtained demonstrate the versatility, robustness, and computational efficiency of the algorithm.

The effective side length expression, $a_{\text {eff }}$, proposed in this study has good accuracy in the range of $2.3<\epsilon_{r}<10.6$ and $0.005<\left(h / \lambda_{d}\right)<0.034$, where $\lambda_{d}$ is the wavelength in the substrate.

It is seen from Tables I-IV that the theoretical results reported by Garg and Long [8],
Kumprasert and Kiranon [14], and Güney [12] are also close to the experimental results. However, the formulae given in this work is simpler than the formulae given elsewhere [8, 12, 14] and also provides the best results.

Because the formula presented in this work has good accuracy and requires no complicated mathematical functions, it can be very useful for the development of fast CAD algorithms. This CAD formula, capable of accurately predicting the resonant frequencies of triangular microstrip antennas, is also very useful to antenna engineers. Using this formula, one can calculate accurately, using a hand calculator, the resonant frequency of triangular patch antennas, without possessing any background knowledge of microstrip antennas. It takes only a few milliseconds to produce the resonant frequencies on a 486 personal computer. Results predicted by the curve-fitting formula obtained using the modified tabu search algorithm agree well with the measured results. The advantages of the formula given here are simplicity and accuracy.

It needs to be emphasized that better and more robust results can be obtained by using the

TABLE III. Comparison of Measured and Calculated Resonant Frequencies of the First Five Modes of an Equilateral Triangular Microstrip Antenna with $a=4.1 \mathrm{~cm}, \varepsilon_{r}=10.5$, and $h=0.07 \mathrm{~cm}$

\begin{tabular}{|c|c|c|c|c|c|c|c|c|c|c|c|c|}
\hline Mode & $\begin{array}{c}f_{m e} \\
{[11]} \\
(\mathrm{MHz})\end{array}$ & $\begin{array}{c}f_{d a} \\
\text { present } \\
\text { method } \\
(\mathrm{MHz})\end{array}$ & $\begin{array}{c}f_{b b} \\
{[1]} \\
(\mathrm{MHz})\end{array}$ & $\begin{array}{c}f_{h j} \\
{[6]} \\
(\mathrm{MHz})\end{array}$ & $\begin{array}{c}f_{g l} \\
{[8]} \\
(\mathrm{MHz})\end{array}$ & $\begin{array}{c}f_{g a} \\
{[9]} \\
(\mathrm{MHz})\end{array}$ & $\begin{array}{c}f_{s d} \\
{[10]} \\
(\mathrm{MHz})\end{array}$ & $\begin{array}{c}f_{c l 1} \\
\text { moment } \\
\text { method [11] } \\
(\mathrm{MHz})\end{array}$ & $\begin{array}{c}f_{c l 2} \\
{[11]} \\
(\mathrm{MHz})\end{array}$ & $\begin{array}{c}f_{g u 1} \\
{[12]} \\
(\mathrm{MHz})\end{array}$ & $\begin{array}{c}f_{k k} \\
{[14]} \\
(\mathrm{MHz})\end{array}$ & $\begin{array}{c}f_{g u 2} \\
{[13]} \\
(\mathrm{MHz})\end{array}$ \\
\hline $\mathrm{TM}_{10}$ & 1519 & 150 & 172 & 198 & 494 & 157 & 14 & & 15 & & 1490 & 1541 \\
\hline $\mathrm{TM}_{11}$ & 2637 & 2600 & 2988 & 2594 & 2588 & 2731 & 2588 & 26 & 2614 & 2617 & 2581 & 2669 \\
\hline $\mathrm{TM}_{20}$ & 2995 & 3002 & 3450 & 2995 & 2989 & 3153 & 2989 & 3025 & 3018 & 3021 & 2980 & 3082 \\
\hline $\mathrm{TM}_{21}$ & 3973 & 3971 & 4564 & 3962 & 3954 & 4172 & 3954 & 4038 & 3993 & 3997 & 3942 & 4077 \\
\hline $\mathrm{TM}_{30}$ & 4439 & 4503 & 5175 & 4493 & 4483 & 4730 & 4483 & 4518 & 4528 & 4532 & 4470 & 4623 \\
\hline
\end{tabular}


TABLE IV. Total Absolute Errors between the Measured and Calculated Resonant Frequencies

\begin{tabular}{|c|c|c|c|c|c|c|c|c|c|c|c|}
\hline & $\begin{array}{c}f_{d a} \\
\text { present } \\
\text { method }\end{array}$ & $\begin{array}{l}f_{b b} \\
{[1]}\end{array}$ & $\begin{array}{l}f_{h j} \\
{[6]}\end{array}$ & $\begin{array}{c}f_{g l} \\
{[8]}\end{array}$ & $\begin{array}{l}f_{g a} \\
{[9]}\end{array}$ & $\begin{array}{c}f_{s d} \\
{[10]}\end{array}$ & $\begin{array}{c}f_{c l 1} \\
\text { moment } \\
\text { method } \\
{[11]}\end{array}$ & $\begin{array}{l}f_{c l 2} \\
{[11]}\end{array}$ & $\begin{array}{l}f_{g u 1} \\
{[12]}\end{array}$ & $\begin{array}{l}f_{k k} \\
{[14]}\end{array}$ & $\begin{array}{l}f_{g u 2} \\
\text { [13] }\end{array}$ \\
\hline $\begin{array}{l}\text { Errors } \\
(\mathrm{MHz})\end{array}$ & 273 & 5124 & 424 & 326 & 1843 & 326 & 472 & 408 & 314 & 349 & 590 \\
\hline
\end{tabular}

modified tabu search algorithm if more experimental data are supplied for the optimization process.

\section{CONCLUSION}

A new, very simple curve-fitting expression for the effective side length was proposed for the resonant frequency of triangular microstrip antennas. This expression was optimally obtained by using a modified tabu search algorithm and is very useful to antenna engineers for accurately predicting the resonant frequencies. The theoretical resonant frequency results calculated by using this new side length expression are in very good agreement with the experimental results available in the literature.

\section{REFERENCES}

1. I. J. Bahl and P. Bhartia, Microstrip Antennas. Artech House, Dedham, MA,1980.

2. J. R. James, P. S. Hall, and C. Wood, Microstrip Antennas - Theory and Design (IEE Electromagnetic Wave Series No. 12). London, Peter Peregrinus Ltd., 1981.

3. J. R. James and P. S. Hall, Handbook of Microstrip Antennas (IEE Electromagnetic Wave Series No. 28) (Vols. 1 and 2). London, Peter Peregrinus Ltd., 1989.

4. Y. T. Lo, S. M. Wright, and M. Davidovitz, "Microstrip Antennas," in K. Chang (ed.), Handbook of Microwave and Optical Components (Vol. 1). New York, Wiley, 1989, pp. 764-889.

5. J. F. Zürcher and F. E. Gardiol, Broadband Patch Antennas. Artech House, Dedham, MA, 1995.

6. J. Helszajn and D. S. James, "Planar Triangular Resonators with Magnetic Walls," IEEE Trans. Microwave Theory Tech., Vol. MTT-26, No. 2, 1978, pp. $95-100$.
7. J. S. Dahele and K. F. Lee, "On the Resonant Frequencies of the Triangular Patch Antenna," IEEE Trans. Ant. Propagat., Vol. AP-35, No. 1, Jan. 1987, pp. 100-101.

8. R. Garg and S. A. Long, "An Improved Formula for the Resonant Frequency of the Triangular Microstrip Patch Antenna," IEEE Trans. Ant. Propagat., Vol. AP-36, 1988, p. 570.

9. X. Gang, "On the Resonant Frequencies of Microstrip Antennas," IEEE Trans. Ant. Propagat., Vol. AP-37, No. 2, 1989, pp. 245-247.

10. R. Singh, A. De, and R. S. Yadava, "Comments on 'An Improved Formula for the Resonant Frequency of the Triangular Microstrip Patch Antenna," IEEE Trans. Ant. Propagat., Vol. AP-39, No. 9, Sept. 1991, pp. 1443-1445.

11. W. Chen, K. F. Lee, and J. S. Dahele, "Theoretical and Experimental Studies of the Resonant Frequencies of the Equilateral Triangular Microstrip Antenna," IEEE Trans. Ant. Propagat., Vol. AP-40, No. 10, Oct. 1992, pp. 1253-1256.

12. K. Güney, "Resonant Frequency of a Triangular Microstrip Antenna," Microwave Opt. Technol. Lett., Vol. 6, No. 9, July 1993, pp. 555-557.

13. K. Güney, "Comments on 'On the Resonant Frequencies of Microstrip Antennas," IEEE Trans. Ant. Propagat., Vol. AP-42, No. 9, Sept. 1994, pp. 1363-1365.

14. N. Kumprasert and W. Kiranon, "Simple and Accurate Formula for the Resonant Frequency of the Equilateral Triangular Microstrip Patch Antenna," IEEE Trans. Ant. Propagat., Vol. AP-42, No. 8, Aug. 1994, pp. 1178-1179.

15. F. Glover, "Tabu Search Part I," ORSA J. Comput., Vol. 1, No. 3, 1989, pp. 190-206.

16. F. Glover, "Tabu Search Part II," ORSA J. Comput., Vol. 2, No. 1, 1990, pp. 4-32.

17. D. Karaboga and A. Kaplan, "Optimising Multivariable Function Using Tabu Search Algorithm," The Tenth International Symposium on Computer and Information Sciences (ISCIS X), Kusadasi, Turkey, Vol. 2, 1995, pp. 793-799.

18. D. Karaboga, "Design of Fuzzy Logic Controllers Using Tabu Search Algorithm," Biennial Conference of the North American Fuzzy Information Processing Society (NAFIPS '96), University 
of California, Berkeley, CA, June 19-22, 1996, pp. 489-491.

19. D. Karaboga and A. Kalinli, "Tuning PID Controller Parameters Using Tabu Search Algorithm," IEEE International Conference on Systems, Man and Cybernetics (IEEE-SMC '96), Beijing, China, October 14-17, 1996, Vol. 1, pp. 134-136.

20. K. Güney, "A New Edge Extension Expression for the Resonant Frequency of Electrically Thick
Rectangular Microstrip Antennas," Int. J. Electron., Vol. 75, No. 4, 1993, pp. 767-770.

21. K. Güney, "Resonant Frequency of a Tunable Rectangular Microstrip Patch Antenna," Microwave Opt. Technol. Lett., Vol. 7, No. 12, Aug. 20, 1994, pp. 581-585.

22. K. Güney, "Resonant Frequency of ElectricallyThick Circular Microstrip Antennas," Int. J. Electron., Vol. 77, No. 3, 1994, pp. 377-386.

\section{BIOGRAPHIES}

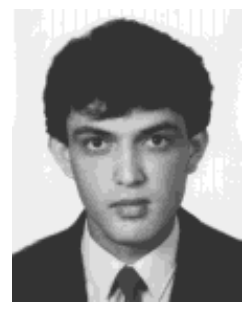

Derviş Karaboğa was born on April 24, 1962, in K. Maraş, Turkey. He received the BS degree from Erciyes University, Kayseri, in 1983, and the MS degree from Istanbul Technical University, in 1988, both in electronic engineering. He received the $\mathrm{PhD}$ degree in systems engineering from the University of Wales College of Cardiff, UK, in 1994. He is now an assistant professor at the Department of Electronic Engineering, Erciyes University. His research interests include modern heuristic optimization techniques (genetic algorithms, tabu searches, and simulated annealing), fuzzy systems, neural networks, digital filters, and antenna design.

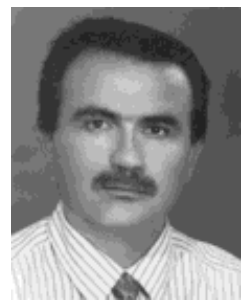

Kerim Güney was born in Isparta, Turkey, on February 28, 1962. He received the BS degree from Erciyes University, Kayseri, in 1983, the MS degree from İstanbul Technical University, in 1988, and the $\mathrm{PhD}$ degree from Erciyes University, in 1991, all in electronic engineering. From 1991 to 1995 he was an assistant professor and now is an associate professor at the Department of Electronic Engineering, Erciyes University, where he is working in the areas of microstrip antennas, antenna synthesis, and optimization techniques.

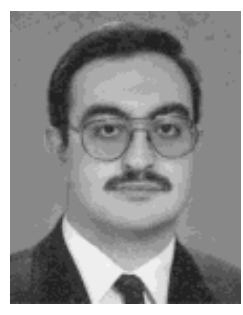

Ahmet Kaplan was born in Kayseri, Turkey, on March 25, 1969. He received the BS degree from Bilkent University, Ankara, in 1992, and the MS degree from Erciyes University, Kayseri, in 1995, both in electronic engineering. Currently, he is a $\mathrm{PhD}$ student and research assistant at the Department of Electronic Engineering, Erciyes University. $\mathrm{He}$ is a student member of the IEEE computer society. His current research activities include optimization techniques, artificial intelligence, and their applications to signal processing and control.

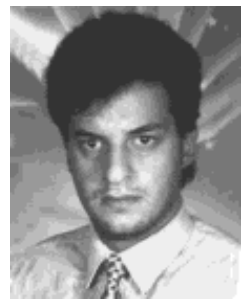

Ali Akdağli was born in Malatya, Turkey, on March 1, 1974. He received the BS degree in electronic engineering from Erciyes University, Kayseri, Turkey, in 1994. $\mathrm{He}$ is now a research assistant and MS student at the Department of Electronic Engineering of Erciyes University, where he is working with microstrip antennas and antenna synthesis. 